KOBE-FHD-94-08

October $20 \quad 1994$

\title{
The Shape of Polarized Gluon Distributions
}

\author{
T. Morii $甘$ \\ Faculty of Human Development, Division of \\ Sciences for Natural Environment \\ and \\ Graduate School of Science and Technology, \\ Kobe University, Nada, Kobe 65\%, Japan \\ S. Tanaka \\ Faculty of Human Development, Division of \\ Sciences for Natural Environment, \\ Kobe University, Nada, Kobe 65\%, Japan \\ and \\ T. Yamanishi \\ Graduate School of Science and Technology, \\ Kobe University, Nada, Kobe 657, Japan
}

\begin{abstract}
The recent high precision SMC data on polarized $\mu p$ scatterings have again confirmed that very little of the proton spin is carried by quarks. To unravel the mystery of the proton spin structure, it is quite important to know the behavior of the polarized gluon distribution. By using the positivity condition of distribution functions together with the unpolarized and polarized experimental data, we restrict the $x$ dependence of the polarized gluon distribution.

\footnotetext{
${ }^{\dagger} \mathrm{E}-\mathrm{mail}$ morii@jpnyitp.bitnet
}

${ }^{\dagger \dagger} \mathrm{E}-\mathrm{mail} \quad$ yamanisi@natura.h.kobe-u.ac.jp
\end{abstract}


Recently, the SMC group[1] at CERN measured the spin-dependent proton structure function $g_{1}^{p}(x)$ more precisely and to the smaller $x$ region up to $x=0.006$ than the previous measurements carried out by the EMC[2]. The experiment indicates that the first moment of $g_{1}^{p}(x)$ increases about $10 \%$ compared to the EMC result, and yet that value is still far from the value predicted by the nonrelativistic quark model and the one from the Ellis--Jaffe sum rule[3]. By combining these SMC data with the experimental data of the neutron $\beta$-decays and hyperon $\beta$-decays, the polarized strange quark density in the proton is derived as follows:

$$
\Delta s=-0.12 \pm 0.04 \pm 0.04
$$

On the other hand, Preparata, Ratcliffe and Soffer have shown that a bound on the value of $\Delta s$ can be obtained by requiring the positivity of distribution functions and assuming the reasonable behavior of the unpolarized s-quark distribution $s(x)$ 田. Quite contrary to the SMC result, they got

$$
|\Delta s| \leq 0.021 \pm 0.001
$$

by using the $s(x)$ derived from the $\nu \mathrm{N}$ deep-inelastic scattering experiments [5]. Furthermore, similar results were obtained by Preparata and Soffer who indicated the following bound on the polarized s-quark density[6]:

$$
|\Delta s| \leq 0.05_{-0.05}^{+0.02}
$$

using CDHS[7] and WA25 data [8]. At first sight, these bounds seem to be contradictory to the SMC data of eq.(1). There might be, however, a compromising solution. If the gluons contribute to the proton spin through the $\mathrm{U}_{A}(1)$ anomaly[9], the left-hand side of eq.(11) should be modified as

$$
\Delta s \rightarrow \Delta s-\frac{\alpha_{S}}{2 \pi} \Delta G
$$


where $\Delta G$ denotes the polarization of gluons. Then the bound of $|\Delta s|$ given by (22) and (3) turns out to be consistent with the SMC data of eq.(信) by taking rather large $\Delta G(\simeq 5-6)$. Namely $\Delta s$ remains small with the cost of large $\Delta G$. Moreover, with this prescription quarks are to carry most of the proton spin and hence one can realize naturally the quark-parton picture. Therefore it is very important to know the magnitude of $\Delta G$ and the $x$ dependence of the polarized gluon distribution $\delta G(x)$, where $\Delta G=\int_{0}^{1} \delta G(x) d x$. So far there have been some interesting studies on the polarized gluon. In literature, various types of the polarized gluon distribution functions have been proposed: some of them have large $\Delta G(\simeq 5-6)$ [10, 11, 12] and others have small $\Delta G(\lesssim 2-3)[11$, 12, 13, 14]. The E581/704 collaboration [15] measured the two-spin asymmetries $A_{L L}^{\pi^{0}}(\stackrel{(-)}{p} p)$ for $\pi^{0}$ productions in polarized $\stackrel{(-)}{p} p$ collisions and concluded, by comparing the measured asymmetries $A_{L L}^{\pi^{0}}(\stackrel{(-)}{p} p)$ with the theoretical predictions by Ramsey et al [12], that the large $\Delta G$ should be ruled out. However, some people [16] have pointed out that the calculations significantly depend on the shape of polarized gluon distribution functions and hence the large $\Delta G$ is not necessarily ruled out but the shape of $\delta G(x)$ is strongly constrained by the E581/704 data.

In this work, we study the $x$ dependence of the polarized gluon distribution $\delta G(x)$. In the previous papers [17, 18], we have proposed a simple model of polarized distributions of quarks and gluons which reproduce the EMC experimental data well. In this model $\Delta s$ was determined to be rather small such as 0.019, which was consistent with the bound of (2) and (3). As for the magnitude of $\Delta G$, we can fix its value to be 5.32 from the experimental data of the integral value of $g_{1}^{p}(x)$. However, as for the $x$ dependence of $\delta G(x)$, nobody knows the exact form of it at present: there remains a number of unknown factors in $\delta G(x)$, which cannot be calculated perturbatively. Here by taking account of the plausible behavior of the 
distribution $\delta G(x)$ near $x \approx 0$ and $x \approx 1$, we assume

$$
\begin{aligned}
\delta G(x) & =G_{+}(x)-G_{-}(x) \\
& =B x^{\gamma}(1-x)^{p}(1+C x)
\end{aligned}
$$

where $G_{+}(x)$ and $G_{-}(x)$ are the gluon distributions with helicity parallel and antiparallel to the proton helicity, respectively. We further assume for simplicity $G_{+}(x) \approx G_{-}(x)$ at large $x$ and take $C=0$. Then there remain two parameters, $\gamma$ and $p . B$ is determined from the normalization, $\Delta G=5.32$. We are interested in the behavior of $\delta G(x)$ under the condition of large $\Delta G(=5.32)$ and study the allowable region of $\gamma$ and $p$. In order to implement this, we require the positivity condition of distribution functions and utilize the recent results of several polarization experiments.

As a preliminary, to examine the behavior of $\delta G(x)$ in eq.(5) for various values of $\gamma$ and $p$, we vary $\gamma$ from -0.9 to 0.3 at intervals of 0.3 while we choose $p$ independently as $5,10,15$, 17 and 20. The results are presented in Fig.1. One can see from this figure that if one takes $\gamma$ smaller, the peak of the distribution is shifted to smaller $x$ and if one takes smaller $p$, the distribution has a broader shoulder.

Now, let us get into the discussion on the restriction of the $x$ dependence of $\delta G(x)$.

(i) First, we consider the positivity condition of distribution functions to restrict $\gamma$ and $p$. As for the unpolarized gluon distribution $G(x)$, we assume

$$
\begin{aligned}
G(x) & =G_{+}(x)+G_{-}(x) \\
& =\frac{A}{x^{\alpha}}(1-x)^{k}
\end{aligned}
$$

like in the case of eq.(5). Since $G_{+}(x)$ and $G_{-}(x)$ are both positive, we obtain from eqs.(5) and (6)

$$
\left|B x^{\gamma}(1-x)^{p}\right| \leq \frac{A}{x^{\alpha}}(1-x)^{k}
$$


From eq.(17) we get

$$
|B| \leq \frac{A}{x^{\alpha+\gamma}}(1-x)^{k-p}
$$

and

$$
|\Delta G| \leq \frac{\Gamma(\gamma+1) \Gamma(p+1) \Gamma(k+3-\alpha)(\alpha+\gamma+p-k)^{\alpha+\gamma+p-k}}{\Gamma(\gamma+p+2) \Gamma(k+1) \Gamma(2-\alpha)(\alpha+\gamma)^{\alpha+\gamma}(p-k)^{p-k}} \int_{0}^{1} x G(x) d x,
$$

To restrict the region of $\gamma$ and $p$ from this inequality (9) with $\Delta G=5.32$, we need to know the value of $\alpha$ and $k$ in $G(x)$ and the intergral value of $x G(x)$ as well. As for the $x$ dependence of $G(x)$, using experimental data of $\mathrm{J} / \psi$ productions for unpolarized muon-nucleon scatterings 19 , 20], we have two possible types of parameterization of $G(x)$ at $Q^{2} \simeq M_{J / \psi}^{2} \mathrm{GeV}^{2}$,

$$
\begin{array}{ll}
\text { Type } A & G(x)=3.35 \frac{1}{x}(1-x)^{5.7}, \\
\text { Type } B & G(x)=2.36 \frac{1}{x^{1.08}}(1-x)^{4.65} .
\end{array}
$$

For Type A, $\alpha$ is taken to be 1 by considering the ordinary Pomeron $\mathrm{P}$, and parametrized so as to fit the data. On the other hand, $\alpha$ is chosen to be 1.08 in Type B which is recently derived from the analysis of the experimental data of the total cross section 21]. The graphs of these two distributions are given in Fig.2, where the intergral values of $x G(x)$ in eqs.(10) and (11) are both normalized to 0.5 in conformity to the experimental data. Inserting these functions into inequality (9) with $\Delta G=5.32$, the allowed regions of $\gamma$ and $p$ are obtained. We have examined (9) for various combinations of $\gamma$ and $p$, and the results are given in Table 1 and Fig.3. In Fig.3, the region below solid or dashed lines is excluded by (9). From this analysis, we conclude that a wide region of $\gamma$ and $p$ which satisfies the SMC data and the positivity condition simultaneously, is allowable with respect to the polarized gluon distribution with large $\Delta G(=5.32)$.

(ii) Second, to restrict further the allowable region of $\gamma$ and $p$, we compare our model 
calculations with the two-spin asymmetries $A_{L L}^{\pi^{0}}(\stackrel{(-)}{p} p)$ for inclusive $\pi^{0}$-productions measured by E581/704 Collaboration using polarized proton (antiproton) beams and polarized proton targets 15. Taking $\delta G(x)$ with the combination of $(\gamma, p)$ which is allowed by the criterion of positivity, we calculate numerically $\left.A_{L L}^{\pi^{0}} \stackrel{(\stackrel{-}{p})}{p}\right)$, where the polarized quark distributions $\delta q_{i}(x)$, which are necessary for the calculation of cross sections for some of subprocesses, are taken from ref.[17]. The results are given in Fig.4. From this figure, some combinations of $\gamma$ and $p$ are excluded. Surviving combinations of $(\gamma, p)$ are shown in Table 2. Comparing the calculations with the experimental data, we have found that $x \delta G(x)$ must have a peak at a smaller $x$ than 0.05 and has to decrease very rapidly with increasing $x$. In short, the experimental data are reproduced well when $\gamma$ is small and $p$ is large, though it is rather difficult to say which one is the best fitting.

(iii) Finally, we look into the spin-dependent structure function of proton $g_{1}^{p}(x)[\mathbb{1}]$ and that of deuteron $g_{1}^{d}(x)$ 222. The merit of considering these parameters is that $g_{1}^{p}(x)$ and $g_{1}^{d}(x)$ do not include undetermined fragmentation functions which were included in $\left.A_{L L}^{\pi^{0}} \stackrel{(-)}{p} p\right)$. Accordingly they are more sensitive to the behavior of $\delta G(x)$. For this case $\gamma$ might be bounded below, while this is not the case for the former two cases. For example, for $\gamma=-0.9$ the calculated values of $x g_{1}^{p}(x)$ seem to deviate from the data for small $x$ regions, $x<0.005$. The new SMC data [1] show a tendency for $g_{1}^{p}(x)$ to increase for small $x, x<0.01$, while the calculated values with $\gamma=-0.9$ keep decreasing for such a small $x$ region. It is expected that if $\gamma$ gets smaller, the discrepancy of $g_{1}^{p}(x)$ between the calculated values and the experimental data would become larger. In addition, for $g_{1}^{d}(x)$ the calculation with $\gamma=-0.9$ does not fit well to the data for $0.01<x<0.05$. The result of calculation using our $\delta q_{i}(x)$ and $\delta G(x)$ with $(\gamma, p)$ surviving the criteria of cases (i) and (ii) is shown in Fig.5 and Table 3. 
In summary, in the models with large $\Delta G(=5.32)$, we have studied the shape of the polarized gluon distribution. By using the positivity condition of distribution functions together with the experimental data on the two-spin asymmetries $\left.A_{L L}^{\pi^{0}} \stackrel{(-)}{p} p\right)$ and the spin-dependent structure functions of $g_{1}^{p}(x)$ and $g_{1}^{d}(x)$, we have restricted the $x$ dependence of $\delta G(x)$ as given in eq.(5). As for the magnitude of $\gamma,-0.6 \lesssim \gamma \lesssim-0.3$ seems favorable in our analysis, and with respect to $p$ we obtain the bound that $p$ should be larger than 15 . In other words, if $\gamma$ and $p$ are fixed in this region, for example, as $\gamma=-0.6$ and $p=17$, one can reproduce all existing data quite successfully. Needless to say, the $\Delta s$ of eq.(1) can be reconciled with the bound of (2) or (3) with large $\Delta G(=5.32)$. However, at present we do not know the theoretical ground on the origin of these values of $\gamma$ and $p$ : in the Regge terminology, the value of $\gamma$ restricted above happens to be closer to the one for unpolarized valence quark distributions rather than for unpolarized gluon distributions 23], and $p$ seems to be inconsistent with the prediction of counting rules[24]. To understand the origin of such $\gamma$ and $p$ is out of scope in this work and needs further investigations. Furthermore, if $\Delta G$ is so large $(\simeq 5-6)$, we are to have an approximate relation $\left\langle L_{Z}\right\rangle_{q+G} \simeq-\Delta G$ from the proton spin sum rule, $\frac{1}{2}=\frac{1}{2} \Delta \Sigma+\Delta G+\left\langle L_{Z}\right\rangle_{q+G}$, where $\frac{1}{2} \Delta \Sigma$ represents the sum of the spin carried by quarks. Unfortunately, nobody knows the underlying physics of it. These are still problems to be solved even though the idea of the $\mathrm{U}_{A}(1)$ anomaly is attractive.

It is informative to comment on another approach which has mentioned to this problem. Recently Brodsky, Burkardt and Schmidt (BBS) [13 have proposed an interesting model of the polarized gluon distribution which incorporates color coherence and counting rule at small and large $x$. At $x \approx 0$, the color coherence argument gives $\delta G(x) / G(x) \approx \frac{x}{3}\left\langle\frac{1}{y}\right\rangle$ with $\left\langle\frac{1}{y}\right\rangle \simeq 3$, where $\left\langle\frac{1}{y}\right\rangle$ presents the first inverse moment of the quark light-cone momentum fraction distributions in the lowest Fock state of the proton, and leads to a relation $\gamma=-\alpha+1$ [13, 14]. Then, 
contrary to our result, $-0.6 \lesssim \gamma \lesssim-0.3$, they have taken $\gamma=0$ by choosing $\alpha=1$ which is an ordinary Pomeron intercept value. In terms of Regge theory, $\gamma=0$ can be interpreted as follows: $\delta G(x)$ at $x \approx 0$ is governed by the $A_{1}$ trajectory. Although the integrated value of $\delta G(x)$ in the BBS model is small such as $\Delta G=0.45$, the model is successful in explaining

the EMC data $g_{1}^{p}(x), g_{1}^{n}(x)$ and $g_{1}^{d}(x)$. In addition, we calculated $\left.A_{L L}^{\pi^{0}} \stackrel{(-)}{p} p\right)$ by using the BBS model and found that the model could reproduce $A_{L L}^{\pi^{0}}(\bar{p} p)$ while the predicted value of $A_{L L}^{\pi^{0}}(p p)$ slightly deviated from the data 18 . The BBS model which has small $\Delta G(=0.45)$ seems to be an alternative to our model which has large $\Delta G(=5.32)$, though in the BBS model the apparent inconsistency between $\Delta s$ of eq.(11) and the bound of (2) or (3) remains to be unsolved.

It is very important to know the behavior of $\delta G(x)$ and $\delta s(x)$ in order to understand the spin structure of a proton. However the polarization experiments are still in their beginning and the form of these functions is not yet clear disappointingly. We hope they will be determined in the forthcoming experiments.

\section{Acknowledgments}

The authors are grateful to N. I. Kochelev and G. Ramsey for their important comments on the behavior of polarized gluons at very small $x$ regions. This work was supported in part by Grant-in-Aid for Scientific Research No.20031370 from the Ministry of Education, Science and Culture, Japan. 


\section{References}

[1] D. Adams et al., SMC Collab., Phys. Lett. B329 (1994) 399.

[2] J. Ashman et al., EMC Collab., Phys. Lett. B206 (1988) 364; Nucl. Phys. B328 (1989) 1.

[3] J. Ellis and R. L. Jaffe, Phys. Rev. D9, (1974) 1444; D10, (1974) 1669.

[4] G. Preparata, P. G. Ratcliffe and J. Soffer, Phys. Lett. B273 (1991) 306.

[5] M. Shaevitz, CCFR Collab., in Proceedings of the Neutrino '90, 1990, CERN.

[6] G. Preparata and J. Soffer, Phys. Rev. Lett. 61 (1988) 1167 ; 62 (1989) 1213 (E).

[7] H. Abramowicz et al., CDHS Collab., Z. Phys. C25 (1984) 29.

[8] D. Allasia et al., WA25 Collab., Z. Phys. C28 (1985) 321.

[9] G. Altarelli and G. G. Ross, Phys. Lett. B212 (1988) 391; R. D. Carlitz, J. C. Collins and A. H. Mueller, Phys. Lett. B214 (1988) 229; A. V. Efremov and O. V. Teryaev, in Proceedings of the International Hadron Symposium, 1988, Bechyně, Czechoslovakia, edited by Fischer et al., (Czechoslovakian Academy of Scinece, Prague, 1989).

[10] G.Altarelli and W.J.Stirling, Part. World 1, (1989) 40; Z. Kunszt, Phys. Lett. B218, (1989) 243; J. Ellis, M. Karliner and C. T. Sachrajda, CERN-TH-5471/89; M. Glück, E. Reya and W. Vogelsang, Phys. Rev. D45, (1992) 2552.

[11] H. Y. Cheng and S. N. Lai, Phys. Rev. D41, (1990) 91.

[12] G. Ramsey and D. Sivers, Phys. Rev. D43, (1991) 2861. 
[13] S. J. Brodsky, M. Burkardt and I. Schmidt, "PERTURBATIVE QCD CONSTRAINTS ON THE SHAPE OF POLARIZED QUARK AND GLUON DISTRIBUTIONS", preprint 6087 (1994).

[14] T. Gehrmann and W. J. Stirling, Durham preprint DTP/94/38 (1994), to be published in Z. Phys. C.

[15] D. L. Adams et al., FNAL E581/704 Collab., Phys. Lett. B261 (1991) 197.

[16] W. Vogelsang and A. Weber, Phys. Rev. D45, (1992) 4069; K. Kobayakawa, T. Morii and T. Yamanishi, Z. Phys. C59, (1993) 251.

[17] K. Kobayakawa, T. Morii, S. Tanaka and T. Yamanishi, Phys. Rev. D46 (1992) 2854.

[18] T. Morii, S. Tanaka and T. Yamanishi, preprint KOBE-FHD-94-01, in Proceedings of the Particle Physics and its Future, 1994, YITP, Japan.

[19] D. Allasia et al., NMC Collab., Phys. Lett. B258 (1991) 493.

[20] J. Ashman et al., EMC Collab., Z. Phys. C56 (1992) 21.

[21] A. Donnachie and P. V. Landshoff, Nucl. Phys. B231 (1984) 189.

[22] B. Adeva et al., SMC Collab., Phys. Lett. B302 (1993) 533.

[23] For example, see R. L. Heimann, Nucl. Phys. B64 (1973) 429; P. Collins: An Introduction to Regge Theory and High-Energy Physics, Cambridge (1977).

[24] V. N. Gribov and L. N. Lipatov, Sov. J. Nucl. Phys. 15 (1972) 438, 675; J. F. Gunion, Phys. Rev. D10, (1974) 242; R. Blankenbecler and S. J. Brodsky, Phys. Rev. D10, (1974) 
2973; B. L. Ioffe, V. A. Khose and L. N. Lipatov: Hard Processes, Amsterdam (1984), Vol.1, p.61. 


\section{Table captions}

Table 1: The various combinations of $\gamma$ and $p$ which we have examined. The circles denote the combinations allowed from (9) whereas the crosses present the ones excluded from (9). The left-side (right-side) table corresponds to Type A (Type B) of $G(x)$.

Table 2: The various combinations of $\gamma$ and $p$ which we have examined. The circles denote the combinations allowed from the $A_{L L}^{\pi^{0}}$ whereas the crosses present the ones excluded from it. The minuses denote the combinations excluded from Table 1. The left-side (right-side) table corresponds to the $A_{L L}^{\pi^{0}}$ for $p p$ collisions ( $\bar{p} p$ collisions).

Table 3: The various combinations of $\gamma$ and $p$ which we have examined. The circles denote the combinations allowed from the spin-dependent structure functions. whereas the crosses present the ones excluded from it. The minuses denote the combinations excluded from Tables 1 and 2. The left-side (right-side) table corresponds to the $g_{1}^{p}(x)$ $\left(g_{1}^{d}(x)\right)$. 


\begin{tabular}{|c|c|c|c|c|c|c|}
\hline & \multicolumn{5}{|c|}{$\mathrm{p}$} \\
\hline & & 5 & 10 & 15 & 17 & 20 \\
\hline \multirow{5}{*}{$\gamma$} & 0.3 & $x$ & $x$ & $\times$ & $x$ & $\times$ \\
\hline & 0.0 & $\bar{x}$ & $\times$ & 0 & 0 & 0 \\
\hline & -0.3 & $x$ & 0 & 0 & 0 & 0 \\
\hline & -0.6 & $x$ & 0 & 0 & 0 & 0 \\
\hline & -0.9 & 0 & 0 & 0 & 0 & 0 \\
\hline
\end{tabular}

\begin{tabular}{|c|c|c|c|c|c|c|}
\hline \multicolumn{2}{|c|}{} & \multicolumn{6}{|c|}{$\mathrm{p}$} \\
\cline { 3 - 7 } \multicolumn{2}{|c|}{} & 5 & 10 & 15 & 17 & 20 \\
\hline \multirow{4}{*}{$\gamma$} & 0.3 & $\times$ & $\times$ & $\times$ & $\times$ & $\times$ \\
\cline { 2 - 7 } & 0.0 & $\times$ & $\times$ & $\times$ & $\bigcirc$ & $\bigcirc$ \\
\cline { 2 - 7 } & -0.3 & $\times$ & $\bigcirc$ & $\bigcirc$ & $\bigcirc$ & $\bigcirc$ \\
\cline { 2 - 7 } & -0.6 & $\times$ & $\bigcirc$ & $\bigcirc$ & $\bigcirc$ & $\bigcirc$ \\
\cline { 2 - 7 } & -0.9 & $\bigcirc$ & $\bigcirc$ & $\bigcirc$ & $\bigcirc$ & $\bigcirc$ \\
\hline
\end{tabular}

Table 1

\begin{tabular}{|c|c|c|c|c|c|c|}
\hline \multicolumn{2}{|c|}{} & \multicolumn{6}{|c|}{$\mathrm{p}$} \\
\cline { 3 - 8 } \multicolumn{2}{|c|}{} & 5 & 10 & 15 & 17 & 20 \\
\hline \multirow{4}{*}{$\gamma$} & 0.3 & - & - & - & - & - \\
\cline { 2 - 7 } & 0.0 & - & - & - & $\times$ & $\bigcirc$ \\
\cline { 2 - 7 } & -0.3 & - & $\times$ & $\times$ & $\bigcirc$ & $\bigcirc$ \\
\cline { 2 - 7 } & -0.6 & - & $\times$ & $\bigcirc$ & $\bigcirc$ & $\bigcirc$ \\
\cline { 2 - 7 } & -0.9 & $\times$ & $\bigcirc$ & $\bigcirc$ & $\bigcirc$ & $\bigcirc$ \\
\hline
\end{tabular}

\begin{tabular}{|c|c|c|c|c|c|c|}
\hline \multicolumn{2}{|c|}{} & \multicolumn{6}{|c|}{$\mathrm{p}$} \\
\cline { 3 - 8 } \multicolumn{2}{|c|}{} & 5 & 10 & 15 & 17 & 20 \\
\hline \multirow{4}{*}{$\gamma$} & 0.3 & - & - & - & - & - \\
\cline { 2 - 7 } & 0.0 & - & - & - & $\bigcirc$ & $\bigcirc$ \\
\cline { 2 - 7 } & -0.3 & - & $\times$ & $\bigcirc$ & $\bigcirc$ & $\bigcirc$ \\
\cline { 2 - 7 } & -0.6 & - & $\bigcirc$ & $\bigcirc$ & $\bigcirc$ & $\bigcirc$ \\
\cline { 2 - 7 } & -0.9 & $\times$ & $\bigcirc$ & $\bigcirc$ & $\bigcirc$ & $\bigcirc$ \\
\hline
\end{tabular}

Table 2

\begin{tabular}{|c|c|c|c|c|c|c|}
\hline \multicolumn{2}{|c|}{} & \multicolumn{6}{|c|}{$\mathrm{p}$} \\
\cline { 3 - 8 } \multicolumn{2}{|c|}{} & 5 & 10 & 15 & 17 & 20 \\
\hline \multirow{4}{*}{$\gamma$} & 0.3 & - & - & - & - & - \\
\cline { 2 - 7 } & 0.0 & - & - & - & - & $\bigcirc$ \\
\cline { 2 - 7 } & -0.3 & - & - & - & $\bigcirc$ & $\bigcirc$ \\
\cline { 2 - 7 } & -0.6 & - & - & $\bigcirc$ & $\bigcirc$ & $\bigcirc$ \\
\cline { 2 - 7 } & -0.9 & - & $\times$ & $\times$ & $\times$ & $\times$ \\
\hline
\end{tabular}

\begin{tabular}{|c|c|c|c|c|c|c|}
\hline \multicolumn{2}{|c|}{} & \multicolumn{6}{|c|}{$\mathrm{p}$} \\
\cline { 3 - 7 } \multicolumn{2}{c|}{} & 5 & 10 & 15 & 17 & 20 \\
\hline \multirow{4}{*}{$\gamma$} & 0.3 & - & - & - & - & - \\
\cline { 2 - 7 } & 0.0 & - & - & - & - & $\bigcirc$ \\
\cline { 2 - 7 } & -0.3 & - & - & - & $\bigcirc$ & $\bigcirc$ \\
\cline { 2 - 7 } & -0.6 & - & - & $\bigcirc$ & $\bigcirc$ & $\bigcirc$ \\
\cline { 2 - 7 } & -0.9 & - & $\times$ & $\times$ & $\times$ & $\times$ \\
\hline
\end{tabular}

Table 3 


\section{Figure captions}

Fig. 1: The $x$ dependence of the spin-dependent gluon distribution function $x \delta G\left(x, Q^{2}\right)$ at $Q^{2}=10 \mathrm{GeV}^{2}$ for various $p(=5-20)$ with (a) $\gamma=0.3$, (b) $\gamma=-0.6$ and (c) $\gamma=-0.9$.

Fig. 2: The parametrization of the gluon distribution functions $x G\left(x, Q^{2}\right)$ at $Q^{2} \approx 10 \mathrm{GeV}^{2}$. The solid (dashed) line denotes Type A (B). The data of opened (closed) circles are taken from [19] $([20])$.

Fig. 3: The allowed region by (9) for $\gamma$ and $p$. The solid (dashed) line corresponds to Type A (B). The region below the lines are excluded.

Fig. 4: The produced $\pi^{0}$ transverse momenta $p_{T}$ dependence of $A_{L L}^{\pi^{0}}(\stackrel{(-)}{p} p)$ for various $p(=$ $5-20$ ) with (a) $\gamma=0$, (b) $\gamma=-0.6$ and (c) $\gamma=-0.9$. Data are taken from [15].

Fig. 5: The $x$ dependence of $x g_{1}^{p}(x)$ and $x g_{1}^{d}(x)$ for various $p(=5-20)$ with $(\mathrm{a}) \gamma=0$, (b) $\gamma=-0.6$ and $(\mathrm{c}) \gamma=-0.9$. The data of $x g_{1}^{p}(x)\left(x g_{1}^{d}(x)\right)$ are taken from [1, 2] ([2]). 
This figure "fig1-1.png" is available in "png" format from: http://arxiv.org/ps/hep-ph/9411228v1 
This figure "fig2-1.png" is available in "png" format from: http://arxiv.org/ps/hep-ph/9411228v1 
This figure "fig3-1.png" is available in "png" format from: http://arxiv.org/ps/hep-ph/9411228v1 
This figure "fig1-2.png" is available in "png" format from: http://arxiv.org/ps/hep-ph/9411228v1 
This figure "fig2-2.png" is available in "png" format from: http://arxiv.org/ps/hep-ph/9411228v1 
This figure "fig3-2.png" is available in "png" format from: http://arxiv.org/ps/hep-ph/9411228v1 
This figure "fig1-3.png" is available in "png" format from: http://arxiv.org/ps/hep-ph/9411228v1 
This figure "fig2-3.png" is available in "png" format from: http://arxiv.org/ps/hep-ph/9411228v1 